\title{
Coverings by few monochromatic pieces - a transition between two Ramsey problems
}

\author{
András Gyárfás \\ Computer and Automation Research Institute \\ Hungarian Academy of Sciences \\ Budapest, P.O. Box 63 \\ Budapest, Hungary, H-1518 \\ gyarfas@sztaki.hu \\ Gábor N. Sárközy* \\ Computer Science Department \\ Worcester Polytechnic Institute \\ Worcester, MA, USA 01609 \\ gsarkozy@cs . wpi.edu \\ and \\ Computer and Automation Research Institute \\ Hungarian Academy of Sciences \\ Budapest, P.O. Box 63 \\ Budapest, Hungary, H-1518 \\ Stanley Selkow \\ Computer Science Department \\ Worcester Polytechnic Institute \\ Worcester, MA, USA 01609 \\ sms@cs.wpi.edu
}

April 4, 2013

\begin{abstract}
11 The typical problem in (generalized) Ramsey theory is to find the order of the largest monochromatic member of a family $\mathcal{F}$ (for example matchings, paths, cycles, connected subgraphs) that must be present in any edge coloring of a complete graph $K_{n}$ with $t$ colors. Another area is to find the minimum number of monochromatic members of $\mathcal{F}$ that partition or cover the vertex set of every edge colored complete graph. Here we propose a problem that connects these areas: for a fixed positive integers $s \leq t$, at least how many vertices can be covered by the vertices of no more than $s$ monochromatic members of $\mathcal{F}$ in every edge coloring of
\end{abstract}

${ }^{*}$ Research supported in part by the National Science Foundation under Grant No. DMS0968699.

${ }^{1}$ Apart from a footnote referring to Pokrovskiy [24], this manuscript was submitted to Graphs and Combinatorics in May, 2011 
$K_{n}$ with $t$ colors. Several problems and conjectures are presented, among them a possible extension of a well-known result of Cockayne and Lorimer on monochromatic matchings for which we prove an initial step: every $t$ coloring of $K_{n}$ contains a $(t-1)$-colored matching of size $k$ provided that

$$
n \geq 2 k+\left\lfloor\frac{k-1}{2^{t-1}-1}\right\rfloor \text {. }
$$

\section{Introduction}

The typical problem in (generalized) Ramsey theory is to find the order of the largest monochromatic member of a family $\mathcal{F}$ (for example matchings, paths, cycles, connected subgraphs) that must be present in any edge coloring of a complete graph $K_{n}$ with $t$ colors. For easier reference these problems are called Ramsey problems in this paper. Another well studied area, we call them cover problems, is to find the minimum number of monochromatic members of $\mathcal{F}$ that partition or cover the vertex set of every edge colored complete graph.

Here we propose a common generalization of Ramsey and cover problems. For a fixed positive integer $s$, at least how many vertices can be covered by the vertices of no more than $s$ monochromatic members of $\mathcal{F}$ in every edge coloring of $K_{n}$ with $t$ colors? A somewhat related problem was proposed by Chung and Liu [4]: for a given graph $G$ and for fixed $s, t$, find the smallest $n$ such that in every $t$-coloring of the edges of $K_{n}$ there is a copy of $G$ colored with at most $s$ colors.

Several problems and conjectures are formulated, among them a possible extension of a well-known result of Cockayne and Lorimer on monochromatic matchings [5]. Our main result (Theorem 2.3) is that every $t$-coloring of $K_{n}$ contains a $(t-1)$-colored matching of size $k$ provided that

$$
n \geq 2 k+\left\lfloor\frac{k-1}{2^{t-1}-1}\right\rfloor \text {. }
$$

This result is sharp. A simple consequence (Corollary 2.4) is that every $t$-colored $K_{2^{t}-2}$ has a perfect matching missing at least one color. This is a special case of a conjecture posed in [1].

\subsection{Path and cycle covers}

As far as path covers in infinite graphs are concerned, Rado 25] has a "perfect" result stated here in an abridged form with its simplified original proof.

Theorem 1.1. The vertex set of any t-colored countable complete graph can be partitioned into finite or one-way infinite monochromatic paths, each of a different color.

Proof. Call a set $C \subseteq\{1, \ldots, t\}$ of $k$ colors, $1 \leq k \leq t$, perfect if there exists a set $\mathcal{P}=\left\{P_{1}, \ldots, P_{k}\right\}$ of $k$ vertex disjoint finite paths $P_{1}=\ldots x_{1}, \ldots, P_{k}=\ldots, x_{k}$ with the following property: $P_{i}$ is monochromatic in color $c_{i}$ and there is an 
infinite set $Y$ of vertices such that $Y$ is disjoint from the paths of $\mathcal{P}$ and for each $i \in\{1, \ldots, k\}$ and for all $y \in Y, x_{i} y$ is colored with $c_{i}$. A perfect color set exists since any color $c_{1}$ present on infinitely many edges of a star incident to vertex $x$ forms such a (one-element) set. Select a perfect set $C$ of $k$ colors so that $k$ is as large as possible $(k \leq t)$, this is witnessed by $\mathcal{P}$ and $Y$. Let $u$ be an arbitrary vertex not covered by $\mathcal{P}$. Consider a color $c$ such that $u y$ has color $c$ for every $y \in Y^{*}$ where $Y^{*} \subseteq Y$, is infinite. It follows from the choice of $k$ that $c \in C$. Now $u$ can be added to the end of the $c$-colored path of $\mathcal{P}$, either directly if $u \in Y$, or through a vertex $v \in Y^{*}$ if $u \notin Y$. The infinite set witnessing the extension is either $Y^{*}$ or $Y^{*} \backslash\{v\}$. Clearly the extensions can be continued to place all vertices of the countable complete graph so that all paths of $\mathcal{P}$ are finite or one-way infinite.

There are several possibilities to "finitize" Theorem 1.1 The 2-color version works perfectly as noted in a footnote in $[8]$.

Proposition 1.2. The vertex set of any 2-colored finite complete graph can be partitioned into monochromatic paths, each of a different color.

Proof. If $P_{1}=\ldots, x_{1}, P_{2}=\ldots, x_{2}$ are red and blue paths and $v$ is uncovered then either $v$ can be placed as the last vertex of one of the paths $P_{i}$ or one of the bypasses $P_{1}, x_{2}, v$ or $P_{2}, x_{1}, v$ extends one monochromatic path (and shortens the other).

Lehel conjectured that Proposition 1.2 remains true if paths are replaced by cycles (where the empty set, one vertex and one edge are accepted as a cycle). Although the existence of a 'near partition' (where the two monochromatic cycles intersect in at most one vertex) follows easily, see 10, it took a long time until this was proved for large $n$ in [21, 1. Recently an elementary proof was found by Bessy and Thomassé [3] that works for all $n$.

Theorem 1.3. (3]) The vertex set of any 2-colored complete graph can be partitioned into two monochromatic cycles of different colors.

In [16] and 6] several possible extensions of Proposition 1.2 were suggested. It turned out that for 3 colors one can not except full partition by distinct colors, the first example of this phenomenon is from Heinrich [18. Recently the asymptotic ratios of monochromatic path and cycle partitions with three distinct colors was obtained in 13 .

Theorem 1.4. ([13]) In every 3 -colored $K_{n}$ at least $\left(\frac{3}{4}-o(1)\right) n$ vertices can be partitioned into monochromatic cycles of distinct colors.

We note that here the asymptotic ratio $\frac{3}{4}$ is best possible. Nevertheless it was conjectured in [6] that Proposition 1.2 and Theorem 1.3 carries over to any number of colors if repetitions of colors are possible.

Conjecture 1.5. ([6]) The vertex set of every t-colored complete graph can be partitioned into $t$ monochromatic cycles.

2 The case of three colors was recently solved in asymptotic sense.

\footnotetext{
${ }^{2}$ Pokrovskiy 24 proved that Conjecture is not quite true for $t=3$
} 
Theorem 1.6. (【13]) In every 3 -colored $K_{n}$ at least $(1-o(1)) n$ vertices can be partitioned into three monochromatic cycles.

The proofs of Theorems 1.4, 1.6 rely on the Regularity Lemma and relaxations of cycles to connected matchings (see Subsection 1.2). The new problem we propose here is the following.

Problem 1.7. Suppose $1 \leq s \leq t$. What is the maximum number of vertices that can be covered by s monochromatic cycles (paths) in every $t$-coloring of the edges of $K_{n}$ ?

We do not have a general conjecture here, not even for the asymptotics (for fixed $s, t$ and large $n$ ). The case $s=1$ is the path Ramsey number where the case $t=3$ ([12]) is the only evidence that perhaps $\frac{n}{t-1}$ is the true asymptotic value $\left(\frac{n}{t}\right.$ is an easy lower bound). The case $s=t$ is in Conjecture 2 ,

The first interesting special case is $t=3, s=2$.

Conjecture 1.8. In any 3 -colored $K_{n}$ there are two vertex disjoint monochromatic paths (cycles) covering at least $\frac{6 n}{7}$ vertices.

A weaker form (for matchings instead of paths) of Conjecture 1.8 follows from Theorem 2.3 below (when $t=3$ ).

\subsection{Connected matchings.}

One technique used recently in many papers (for example [7, 12, [15]) in Ramsey and in covering problems related to paths or cycles is to replace the paths or cycles by a simpler structure, monochromatic connected matchings, and rely on the Regularity and Blow-up Lemmas to create paths or cycles from them. A connected monochromatic matching means that all edges of the matching are in the same component of the subgraph induced by the edges in the color of the matching.

Thus a natural step towards proving an asymptotic (or sharp for large enough $n$ ) version of Conjecture 1.8 would be to prove it for connected matchings. However, in the problem mentioned above we are a step behind, we could only prove Conjecture 1.8 for matchings (without the connectivity condition). A logical plan is to treat connected pieces and matchings separately, this is done in Subsection 1.3 and in Section 2 .

\subsection{Covers by connected components}

Since every connected component contains a spanning tree of the component, we use here the somewhat simpler tree language. A special case of a conjecture attributed to Ryser, (appearing in his student, Henderson's thesis [19]) states that every intersecting $t$-partite hypergraph has a transversal of at most $t-1$ elements. Using the dual of the hypergraph of monochromatic components in a $t$-coloring of complete graphs, one can easily see that the following form of the conjecture (introduced in [9]) is equivalent. 
Conjecture 1.9. In every t-coloring of the edges of a complete graph, the vertex set can be covered by the vertices of at most $t-1$ monochromatic trees.

If Conjecture 1.9 is true then an easy averaging argument would easily extend it as follows.

Conjecture 1.10. For every $1 \leq s \leq t-1$ and for every $t$-coloring of $K_{n}$ at least $\frac{n s}{t-1}$ vertices can be covered by the vertices of at most $s$ monochromatic trees.

Since Conjecture 1.9 is known to be true for $2 \leq t \leq 5$, Conjecture 1.10 is true for $1 \leq s \leq t \leq 5$. Also, the case $s=1$ is known for arbitrary $t$ (originally in [9, 17] is a recent survey). Perhaps a good test case is to try to prove Conjecture 1.10 for $s=2$ (and for general $t$ ).

Since Ryser's conjecture is extended further in [6] by changing cover to partition in Conjecture 1.9, one may perhaps even require partition in Conjecture 1.10 as well.

\subsection{Covers by copies of a fixed graph}

It seems that to find the percentage of vertices that can be covered by monochromatic copies of a fixed graph $H$ having at most $s$ colors is a difficult problem. Indeed, even the case when $H$ is a single edge seems difficult. However, somewhat surprisingly, for any fixed connected non-bipartite graph $H$ and for any fixed $t \geq 3$ and fixed $s \leq t$, the percentage of vertices of $K_{n}$ that can be covered by vertex disjoint monochromatic copies having at most $s$ colors can be rather well approximated. In fact, the following theorem can be easily obtained from the results of 22. Let $R_{t}(H)$ denote the smallest integer $m$ such that in every $t$-coloring of the edges of $K_{n}$ there is a monochromatic copy of $H$.

Theorem 1.11. Suppose that $t \geq 3,1 \leq s \leq t$ and $H$ is a connected nonbipartite graph. Then in every $t$-coloring of the edges of $K_{n}$, at least $\frac{s\left(n-R_{t}(H)\right)}{t}$ vertices can be covered by vertex disjoint monochromatic copies of $H$ using at most $s$ colors. On the other hand, for any $n$ that is divisible by $t$, the edges of $K_{n}$ can be $t$-colored so that at most $\frac{s n}{t}$ vertices can be covered by vertex disjoint monochromatic copies of $H$ having at most $s$ colors.

Proof. The first part follows by selecting successively monochromatic copies of $H$, removing after each step the part covered so far. Clearly, the process stops only when less than $R_{t}(H)$ vertices remain. Then, an obvious averaging argument gives that the copies in $s$ suitable colors cover the claimed quantity.

The second part follows from the following construction. Partition $V\left(K_{n}\right)$ into $t$ equal parts and color the edges within the parts with $t$ different colors, say within part $i$ every edge gets color $i$. The crossing edges (going from one part to another) are all colored with the same color between any fixed pair of parts. There are two rules. On one hand, crossing edges of color $i$ cannot be incident to part $i$. On the other hand, the union of crossing edges of color $i$ should span a bipartite graph. It is easy to see that these rules can be easily guaranteed for 
$t \geq 3$ (and impossible to meet for $t=2$ ). Because $H$ is connected, not bipartite and crossing edges of color $i$ are not adjacent to part $i$, each monochromatic copy of $H$ must be completely within a part. Therefore copies of $H$ having at most $s$ colors are covered by the at most $s$ parts, proving the second statement of the theorem.

\section{Covers by matchings - how to generalize Cock- ayne - Lorimer theorem?}

Here we return to the most basic case, when we want to cover by copies of an edge, i.e. by matchings. A matching in a $t$-colored complete graph is called an $s$-colored matching if at most $s$ colors are used on its edges. To describe easily certain $t$-colorings of $K_{n}$ we need, consider partition vectors with $t$ positive integer coordinates whose sum is equal to $n$. Assume that $V\left(K_{n}\right)=\{1,2, \ldots, n\}$. Then $\left[p_{1}, p_{2}, \ldots, p_{t}\right]$ represents the coloring obtained by partitioning $V\left(K_{n}\right)$ into parts $A_{i}$ so that $\left|A_{i}\right|=p_{i}$ for $i=1,2, \ldots, t$ and the color of any edge $e=(x, y)$ is the minimum $j$ for which $\{x, y\}$ has non-empty intersection with $A_{j}$.

Problem 2.1. Suppose $1 \leq s \leq t$. What is the size of the largest $s$-colored matching that can be found in every t-coloring of the edges of $K_{n}$ ?

The Ramsey problem, the case $s=1$ in Problem 2.1, was completely answered by Cockayne and Lorimer [5]. Here we state its diagonal case only.

Theorem 2.2. ([5]) Assume $n \geq(t+1) p+2$ and $K_{n}$ is arbitrarily $t$-colored. Then there is a monochromatic matching of size $p+1$.

Observe that Theorem 2.2 is sharp, the coloring $[p, p, \ldots, p, 2 p+1]$ of $K_{(t+1) p+1}$ does not contain a monochromatic matching with $p+1$ edges.

Notice that the case $s=t$ of Problem 2.1 is trivial, any perfect (or nearperfect if $n$ is odd) matching is obviously optimal. In this paper we settle the case $s=t-1$, by showing that the extremal coloring is close to the coloring $\left[p, 2 p, 4 p, \ldots, 2^{t-1} p\right]$. More precisely we prove the following.

Theorem 2.3. Every $t$-coloring of $K_{n}$ contains a $(t-1)$-colored matching of size $k$ provided that

$$
n \geq 2 k+\left\lfloor\frac{(k-1)}{2^{t-1}-1}\right\rfloor .
$$

This is sharp for every $t \geq 2, k \geq 1$.

In case of $t=3$ Theorem 2.3 gives Conjecture 1.8 in a weaker form. Noting that for $k<2^{t-1}$ the second term is zero in Theorem 2.3. we get the following.

Corollary 2.4. Every t-colored $K_{2^{t}-2}$ has a perfect matching missing at least one color. 
We note here that for $t=2,3,4$ there are results stronger than Theorem 2.3 . Namely, not only a $(t-1)$-colored matching of size $k$ can be guaranteed, but a $(t-1)$-colored path on $2 k$ vertices. For $t=2$ this is a well-known result [8], for $t=3$ it was proved in [23] and for $t=4$ in [20]. In fact, it was conjectured in [20] that Theorem 2.3 holds also if the matching of size $k$ is replaced by $P_{2 k}$.

For the case $t=4, s=2$ we suspect that the extremal coloring is essentially $[p, p, 2 p, 4 p]$. That leads to

Conjecture 2.5. If $n \geq\left\lfloor\frac{8 k-2}{3}\right\rfloor$ then every 4 -coloring of $K_{n}$ contains a 2 -colored matching of size $k$.

For the case $s=2, t=5$, the coloring $[p, p, p, 2 p, 4 p]$ and the coloring $[p, p, p, p, 2 p]$ that belongs to Theorem 2.2 give essentially the same parameters so we do not risk a conjecture here. Moreover, for $s=2, t=6$ the latter coloring $[p, p, p, p, p, 2 p]$ is better than $[p, p, p, p, 2 p, 4 p]$. This leads to the dilemma whether there are better coloring in this case or $[p, p, p, p, p, 2 p]$ is the extremal one? The latter possibility would be similar to the phenomenon discussed in Subsection 1.4, saying vaguely that in a 6-colored complete graph the size of the largest 2-matching is twice the size of the largest monochromatic matching.

\section{Large $(t-1)$-colored matchings in $t$-colored complete graphs.}

Here we prove Theorem 2.3. To show that it is sharp, set $N=2 k-1+$ $\left\lfloor\frac{(k-1)}{2^{t-1}-1}\right\rfloor=2 k-1+p$, where $p=\left\lfloor\frac{N}{2^{t}-1}\right\rfloor=\left\lfloor\frac{k-1}{2^{t-1}-1}\right\rfloor$. Consider the coloring $\left[p, 2 p, 4 p, \ldots, 2^{t-2} p, q\right]$ of $K_{N}$ with $q=N-\left(2^{t-1}-1\right) p$.

If a matching in this coloring misses color $j \neq t$ then it misses at least $2^{j-1} p-\sum_{i<j} 2^{i-1} p=p$ vertices from the vertex set to which color class $j$ is incident to. Thus at most $N-p=2 k-1$ vertices are covered by this matching so its size is smaller than $k$. A matching that misses color $t$ has at most $\sum_{i<t} 2^{i-1} p=\left(2^{t-1}-1\right) p \leq k-1$ edges.

To prove the upper bound, consider a $t$-colored $K_{n}$ where $n=2 k+\left\lfloor\frac{(k-1)}{2^{t-1}-1}\right\rfloor=$ $2 k+p$. Set $V=V\left(K_{n}\right)$, let $G_{i}$ denote the subgraph of $K_{n}$ with vertex set $V$ and containing edges of colors different from color $i, 1 \leq i \leq t$. We are going to show that for at least one $i, G_{i}$ has a matching of size $k$. The proof is indirect: if the maximum matching of $G_{i}, \nu\left(G_{i}\right)$, is at most $k-1$ for each $i$, then for the deficiency of $G_{i}$, def $\left(G_{i}\right)$, defined as the the number of vertices uncovered by any maximum matching of $G_{i}$, we have

$$
\operatorname{def}\left(G_{i}\right) \geq n-2 \nu\left(G_{i}\right) \geq 2 k+p-2(k-1)=p+2 .
$$

We apply the following well-known result, where $c_{o}(G)$ is the number of odd components of $G$.

Theorem 3.1. (Berge formula) $\operatorname{def}(G)=\max \left\{c_{o}(V(G) \backslash X)-|X|: X \subset\right.$ $V(G)\}$. 
Thus, for each $i$, there is a set $X_{i} \subset V$ such that

$$
c_{o}\left(V \backslash X_{i}\right) \geq\left|X_{i}\right|+p+2 .
$$

Assume w.l.o.g that $\left|X_{1}\right| \leq \ldots, \leq\left|X_{t}\right|$ and observe that the edges between connected components of $G_{i}$ in $V \backslash X_{i}$ are all colored with color $i$. Let $C_{1}^{1}, \ldots, C_{m_{1}}^{1}$ be the vertex sets of the connected components of $G_{1}$ in $V \backslash X_{1}$, from (11) we have $m_{1} \geq\left|X_{1}\right|+p+2$.

Lemma 3.2. There is an index $l \in\left\{1,2, \ldots, m_{1}\right\}$, say $l=1$, such that for every $j>1, \cup_{i \neq 1} C_{i}^{1} \subset X_{j}$.

Proof. Suppose that $v, w \notin X_{2}$ where $v \in C_{q}^{1}, w \in C_{r}^{1}$ and $q \neq r$. This implies that the edges of color 1 form a complete multipartite graph $M$ on $V \backslash\left(X_{1} \cup X_{2}\right)$ with at least two partite classes. Therefore all vertices of $M$ must be in the same connected component of $G_{2}$ in $V \backslash X_{2}$. Thus $G_{2}$ has at most $1+\left|X_{1}\right| \leq 1+\left|X_{2}\right|<\left|X_{2}\right|+p+2$ odd components in $V \backslash X_{2}$, contradicting (11). Thus $X_{2}$ must cover all but at most one among the $C_{i}^{1}$-s, say $C_{1}^{1}$ can be uncovered.

Next we show that for all $j \geq 2$, we have $\cup_{i \neq 1} C_{i}^{1} \subset X_{j}$. We have seen this for $j=2$, so assume $j>2$. The argument of the previous paragraph gives that $X_{j}$ covers all but one $C_{i}^{1}$, say the exceptional one is $C_{l}^{1}$. Suppose $l \neq 1$, say $l=2$. The inequality $\left|X_{2}\right| \leq\left|X_{j}\right|$ implies $\left|X_{2} \backslash X_{j}\right| \leq\left|X_{j} \backslash X_{2}\right|$ and from this

$$
\left|X_{1} \cap\left(X_{2} \backslash X_{j}\right)\right|+\left|C_{2}^{1} \backslash X_{j}\right| \leq\left|X_{1} \cap\left(X_{j} \backslash X_{2}\right)\right|+\left|C_{1}^{1} \backslash X_{2}\right| .
$$

On the other hand, using (1),

$$
\begin{aligned}
\left|V \backslash X_{j}\right| & =\left|X_{1} \cap\left(X_{2} \backslash X_{j}\right)\right|+\left|X_{1} \backslash\left(X_{2} \cup X_{j}\right)\right|+\left|C_{2}^{1} \backslash X_{j}\right| \geq c_{o}\left(V \backslash X_{j}\right) \geq\left|X_{j}\right|+p+2 \\
& \geq\left|X_{1} \cap\left(X_{j} \backslash X_{2}\right)\right|+\left|C_{1}^{1} \backslash X_{2}\right|+\left|X_{j} \cap C_{2}^{1}\right|+\left|\cup_{i \geq 3} C_{i}^{1}\right|+p+2
\end{aligned}
$$

which can be rearranged as

$$
\begin{gathered}
\left(\left|X_{1} \cap\left(X_{2} \backslash X_{j}\right)\right|+\left|C_{2}^{1} \backslash X_{j}\right|\right)-\left(\left|X_{1} \cap\left(X_{j} \backslash X_{2}\right)\right|+\left|C_{1}^{1} \backslash X_{2}\right|\right)+\left|X_{1} \backslash\left(X_{2} \cup X_{j}\right)\right| \geq \\
\geq\left|X_{j} \cap C_{2}^{1}\right|+\left|\cup_{i \geq 3} C_{i}^{1}\right|+p+2 .
\end{gathered}
$$

Note that from (2) the left hand side of (3) is at most $\left|\left(X_{2} \cup X_{j}\right)\right|$. Thus from (3) we get

$\left|X_{1}\right| \geq\left|X_{1} \backslash\left(X_{2} \cup X_{j}\right)\right| \geq\left|X_{j} \cap C_{2}^{1}\right|+\left|\cup_{i \geq 3} C_{i}^{1}\right|+p+2 \geq m_{1}-2+p+2=m_{1}+p$

and this contradicts $m_{1} \geq\left|X_{1}\right|+p+2$ and finishes the proof of the lemma.

Call $\operatorname{Ker}_{1}=C_{2}^{1} \cup \ldots \cup C_{m_{1}}^{1}$ the first kernel. With this notation Lemma 3.2 claims that each $X_{j}$ with $j>1$ contains $K e r_{1}$. We may iterate Lemma 3.2 to define the set $K_{e r}=C_{2}^{i} \cup \ldots \cup C_{m_{i}}^{i}$, the $i$-th kernel, so that each $X_{j}$ with $j>i$ contains $K e r_{i}$. Furthermore, these kernels are disjoint, since $X_{i+1}$ contains $K e r_{i}$, but $K e r_{i+1}$ is contained in $V \backslash X_{i+1}$. This implies that we have the following recursion on the sizes of the $X_{i}$ 's. 
Claim 1. For every $2 \leq i \leq t$ we have

$$
\left|X_{i}\right| \geq\left|X_{1}\right|+\ldots+\left|X_{i-1}\right|+(i-1)(p+1) .
$$

Indeed, $X_{i}$ contains all the disjoint kernels $K e r_{1}, \ldots, K e r_{i-1}$ and thus using (11) we get

$$
\left|X_{i}\right| \geq \sum_{j=1}^{i-1}\left|\operatorname{Ker}_{j}\right| \geq \sum_{j=1}^{i-1}\left(c_{0}\left(V \backslash X_{j}\right)-1\right) \geq \sum_{j=1}^{i-1}\left(\left|X_{j}\right|+p+1\right),
$$

as desired.

Claim 1 implies easily by induction the following

$$
\left|X_{i}\right| \geq\left(2^{i-1}-1\right)(p+1) .
$$

But then, since the kernels are disjoint, using (11) again we get the following contradiction

$$
\begin{aligned}
& n \geq \sum_{i=1}^{t}\left|K e r_{i}\right| \geq \sum_{i=1}^{t}\left(c_{0}\left(V \backslash X_{i}\right)-1\right) \geq \sum_{i=1}^{t}\left(\left|X_{i}\right|+p+1\right)= \\
& =\sum_{i=1}^{t}\left|X_{i}\right|+t(p+1) \geq \sum_{i=1}^{t} 2^{i-1}(p+1)=\left(2^{t}-1\right)(p+1)>n .
\end{aligned}
$$

Here for the last inequality we have to check

$$
\left(2^{t}-1\right)(p+1) \geq n+1=2 k+p+1 .
$$

This is equivalent to

$$
p+1 \geq \frac{k}{2^{t-1}-1},
$$

which is always true for our choice $p=\left\lfloor\frac{k-1}{2^{t-1}-1}\right\rfloor$. Indeed,

$$
\left\lfloor\frac{k-1}{2^{t-1}-1}\right\rfloor=\left\lfloor\frac{k}{2^{t-1}-1}\right\rfloor
$$

(and so (5) is trivially true) for all cases except when $\frac{k}{2^{t-1}-1}$ is an integer, but (5) is true in this case as well, finishing the proof of Theorem 2.3.

\section{References}

[1] P. Allen, Covering two-edge-coloured complete graphs with two disjoint monochromatic cycles, Combinatorics, Probability and Computing, 17 (2008), 471-486.

[2] S. A. Burr, P. Erdős, J. H. Spencer, Ramsey theorems for multiple copies graphs, Transactions of the American Mathematical Society 209 (1975), pp. 87-99. 
[3] S. Bessy, S. Thomassé, Partitioning a graph into a cycle and an anticycle, a proof of Lehel's conjecture, Journal of Combinatorial Theory B., 100 (2009), 176-180.

[4] K.M. Chung, C.L. Liu, A generalization of Ramsey theory for graphs, Discrete Mathematics $\mathbf{2}$ (1978) 117-127.

[5] E. J. Cockayne, P. J. Lorimer, The Ramsey number for stripes, J. Austral. Math. Soc. 19 (1975), pp. 252-256.

[6] P. Erdős, A. Gyárfás, L. Pyber, Vertex coverings by monochromatic cycles and trees, Journal of Combinatorial Theory B 51 (1991) 90-95.

[7] A. Figaj, T. Luczak, The Ramsey number for a triple of long even cycles, to appear in the Journal of Combinatorial Theory, Ser. B 97 (2007) 584-596.

[8] L. Gerencsér, A. Gyárfás, On Ramsey type problems, Ann. Univ. Sci. Eötvös, Budapest 10 (1967) 167 - 170.

[9] A. Gyárfás, Partition coverings and blocking sets in hypergraphs (in Hungarian) Communications of the Computer and Automation Institute of the Hungarian Academy of Sciences 71 (1977) 62 pp.

[10] A. Gyárfás, Vertex coverings by monochromatic paths and cycles, Journal of Graph Theory 7. (1983)131-135.

[11] A. Gyárfás, Large matchings with few colors - a problem for 'Emléktábla' workshop, manuscript, 2010.

[12] A. Gyárfás, M. Ruszinkó, G. N. Sárközy, E. Szemerédi, Three-color Ramsey numbers for paths, Combinatorica, 27 (2007), pp. 35-69.

[13] A. Gyárfás, M. Ruszinkó, G. N. Sárközy, E. Szemerédi, Partitioning 3colored complete graphs into three monochromatic cycles, Electronic J. of Combinatorics 18(2011) N.53.

[14] A. Gyárfás, G. N. Sárközy, E. Szemerédi, The Ramsey number of diamond matchings and loose cycles in hypergraphs, Electronic Journal of Combinatorics, 15 (2008), R126.

[15] A. Gyárfás, M. Ruszinkó, G. N. Sárközy, E. Szemerédi, One-sided coverings of complete bipartite graphs, in Algorithms and Combinatorics 26, Topics in Discrete Mathematics, 133-144.

[16] A. Gyárfás, Monochromatic path covers, Congressus Numerantium 109 (1995), 201-202.

[17] A. Gyárfás, Large monochromatic components in edge colorings of graphs - a survey, 'Ramsey Theory Yesterday, Today and Tomorrow, DIMACS workshop 2008 To appear in 'Progress in Mathematics' series. 
[18] Kathy Heinrich, personal communication, 1994.

[19] J. R. Henderson, Permutation Decomposition of (0-1)-Matrices and Decomposition Transversals, Ph.D. thesis, Caltech, 1971.

[20] A. Khamseh, G. R. Omidi, A generalization of Ramsey theory for linear forests, manuscript submitted in 2010.

[21] T. Łuczak, V. Rödl, E. Szemerédi, Partitioning two-colored complete graphs into two monochromatic cycles, Combinatorics, Probability and Computing, 7 (1998), 423-436.

[22] P.J. Lorimer, R.J. Segedin, Ramsey numbers for multiple copies of complete graphs, Journal of Graph Theory, 2 (1978) 89-91.

[23] R.Meenakshi, P.S. Sundararaghavan, Generalized Ramsey numbers for paths in 2-chromatic graphs, Internat. J. Math. and Math. Sci. 9 (1986) $273-276$.

[24] A. Pokrovskiy, Partitioning edge-coloured complete graphs into monochromatic cycles and paths, arXiv:12.05.5492v1

[25] R. Rado, Monochromatic paths in graphs, Annals of Discrete Mathematics 3 (1987) 89-91. 